\title{
A fermented formula in pre-term infants: clinical tolerance, gut microbiota, down-regulation of faecal calprotectin and up-regulation of faecal secretory IgA
}

\author{
Florence Campeotto ${ }^{1,2}$, Antonia Suau ${ }^{3}$, Nathalie Kapel ${ }^{2,4}$, Fabien Magne ${ }^{3}$, Vivian Viallon ${ }^{5}$, \\ Laurent Ferraris ${ }^{2}$, Anne-Judith Waligora-Dupriet ${ }^{2}$, Pascale Soulaines ${ }^{1}$, Bernard Leroux ${ }^{6}$, Nicolas Kalach ${ }^{1}$, \\ Christophe Dupont ${ }^{1,7 *}$ and Marie-José Butel ${ }^{2}$ \\ ${ }^{1}$ Néonatologie et Gastroentérologie pédiatrique, APHP, Groupe Hospitalier Cochin-Saint Vincent de Paul, Université Paris \\ Descartes, 78-82 Avenue Denfert-Rochereau, 75014 Paris, France \\ ${ }^{2}$ Ecosystème intestinal, probiotiques, antibiotiques (EA 4065), Université Paris Descartes, 4 Avenue de l'Observatoire, \\ 75006 Paris, France \\ ${ }^{3}$ Laboratoire de Biologie, EA 3199, Conservatoire National des Arts et Métiers, 2 rue Conté, 75003 Paris, France \\ ${ }^{4}$ Coprologie fonctionnelle, APHP, Groupe hospitalier Pitié-Salpêtrière, 47-83 boulevard de l'Hôpital, 75013 Paris, France \\ ${ }^{5}$ Biostatistique, APHP, Groupe Hospitalier Cochin-Port Royal, 27 rue du Faubourg St Jacques, 75014 Paris, France \\ ${ }^{6}$ Néonatologie, Unité Mère-Enfant Alix de Champagne, 23 rue des Moulins, 51000 Reims, France \\ ${ }^{7}$ Service d'Explorations Fonctionnelles Digestives Pédiatriques, Gastroentérologie et Allergie alimentaire, Hôpital \\ Necker-Enfants Malades, 149 rue de Sèvres, 75015 Paris, France
}

(Received 22 July 2010 - Revised 3 December 2010 - Accepted 6 December 2010 - First published online 22 March 2011)

\begin{abstract}
Intestinal bacterial colonisation in pre-term infants is delayed compared with full-term infants, leading to an increased risk of gastrointestinal disease. Modulation of colonisation through dietary supplementation with probiotics or prebiotics could decrease such a risk. The present study evaluated clinical tolerance, the effects on gut microbiota, and inflammatory and immunological mucosal responses to an infant formula adapted for pre-term infants that included in its manufacturing process a fermentation step with two probiotic strains, Bifidobacterium breve C50 and Streptococcus thermophilus 065, inactivated by heat at the end of the process. A total of fifty-eight infants (gestational age: 30-35 weeks), fed either the fermented pre-term formula or a standard pre-term formula, were followed up during their hospital stay. Clinical tolerance, faecal microbiota using a culture and a culture-independent method (temporal temperature gel electrophoresis), faecal calprotectin and secretory IgA were analysed weekly. No difference was observed regarding anthropometric data and digestive tolerance, except for abdominal distension, the incidence of which was lower in infants fed the fermented formula for 2 weeks. Bacterial colonisation was not modified by the type of feeding, particularly for bifidobacteria. Faecal calprotectin was significantly lower in infants fed the fermented formula for 2 weeks, and secretory IgA increased with both mother's milk and the fermented formula. The fermented formula was well tolerated and did not significantly modulate the bacterial colonisation but had benefits on inflammatory and immune markers, which might be related to some features of gastrointestinal tolerance.
\end{abstract}

Key words: Pre-term infants: Fermented formula: Gut microbiota: Calprotectin: Secretory IgA

Providing optimal enteral nutrition to pre-term infants is one of the major clinical challenges facing neonatologists, and, more recently, interest has turned to the role of nutrition in the primary prevention of diseases. Several clinical studies have investigated the potential benefit of modulating the gut microbiota in pre-term infants - who display a delayed and impaired gut bacterial colonisation ${ }^{(1)}$ - through supplementation with probiotics and/or prebiotics ${ }^{(2,3)}$. The most promising benefit of enteral supplementation with probiotics - defined as live microbial supplements that potentially provide benefits to the host - is the prevention of necrotising enterocolitis (NEC), as demonstrated by the most recent

Abbreviations: FPF, fermented pre-term formula; GA, gestational age; NEC, necrotising enterocolitis; PF, pre-term formula; SIgA, secretory IgA; TTGE, temporal temperature gel electrophoresis.

The present study was presented at the 9th Biennial Congress of the Anaerobe Society of the Americas, 24-27 June 2008, Long Beach, CA, USA.

* Corresponding author: Professeur C. Dupont, fax +33 171196120, email christophe.dupont@nck.aphp.fr

† Present address: APHP, Hôpital Necker-Enfants Malades, Université Paris Descartes, 149 rue de Sèvres, 75015 Paris, France. 
meta-analyses that showed the very significant benefits of probiotic supplements ${ }^{(4,5)}$. However, according to the latest comment by the European Society of Pediatric Gastroenterology, Hepatology and Nutrition Committee on Nutrition, there is not enough available evidence on the safety of probiotics in pre-term infants ${ }^{(6)}$. Although infrequent, systemic translocation of probiotics has been reported ${ }^{(7,8)}$, raising some concerns about this side effect in high-risk groups of low- and very-low-birth-weight infants who are characterised by high intestinal permeability.

Prebiotics are non-digestible oligosaccharides leading to specific changes in the composition and/or activity of beneficial gut microbiota ${ }^{(9)}$. The bifidogenic effect of the infant formula supplemented with prebiotics has been demonstrated and is thought to be beneficial ${ }^{(3,10)}$. In pre-term infants, only a few studies have shown that oligosaccharide supplementation increased bifidobacterial and lactobacilli levels ${ }^{(11)}$, reducing the presence of clinically relevant pathogens in the gut microbiota. Furthermore, the clinical benefits of these supplementations were poorly demonstrated ${ }^{(2,11)}$, although reduced stool viscosity and accelerated gastrointestinal transport were observed in pre-term infants fed a prebiotic formula ${ }^{(12)}$, and in a recent study, a trend has been found towards a lower incidence of serious infectious morbidity in pre-term infants receiving enteral supplementation of a prebiotic mixture ${ }^{(13)}$.

An alternative option is to use formulas fermented with lactic acid-producing bacteria during the production process that are subsequently inactivated by heat or other means at the end of the process ${ }^{(14)}$. This leads to a probiotic/prebiotic activity that is probably related to both the production of active bacterial metabolites such as transoligosaccharides and the presence of bacterial components such as cell membrane and DNA ${ }^{(15,16)}$. A formula manufactured with Bifidobacterium breve and Streptococcus thermophilus (Blédina, Villefranchesur-Saône, France) has shown some benefits in full-term infants, including a bifidogenic effect ${ }^{(14,17)}$, reduced diarrhoeal severity ${ }^{(18)}$, increased specific intestinal antibody response ${ }^{(17)}$ and increased thymus size ${ }^{(19)}$. This kind of formula has never been tested in pre-term infants. The present study aimed at investigating clinical tolerance and the effect on the gut microbiota of early feeding of pre-term infants with this fermented formula adapted for these infants. TNF- $\alpha$, calprotectin and secretory IgA (SIgA) were also monitored.

\section{Subjects and methods}

\section{Subjects and trial design}

The trial was carried out in two neonatal units, St Vincent de Paul hospital (Paris) and Alix de Champagne hospital (Reims), from December 2002 to February 2005. The study protocol was conducted according to the guidelines laid down in the Declaration of Helsinki, and all procedures involving human patients were approved by the local ethics committee (CPP Ile de France III, no. 1977/06-05-02). Pre-term infants with a gestational age (GA) less than 35 weeks and whose mother chose formula feeding were enrolled during their first $3 \mathrm{~d}$ of life; enrolment ended at the hospital discharge.
Parents provided informed written consent. Infants were randomly assigned to receive either a formula adapted for pre-term infants (PF), as a control, or the formula containing heat-killed ferments (FPF). Doctors, nurses, research staff and parents were blinded to which formula the infant was given. Newborns whose parents did not provide informed consent or who had malformations or metabolic diseases were excluded from the study. In St Vincent de Paul hospital, due to a high prevalence of mothers wishing to breast-feed, neonates whose mothers decided to attempt to breast-feed could be enrolled, resulting for some infants having had a mixed diet.

The intent-to-treat population consisted of all randomised infants, and the per-protocol population was defined as infants for whom at least two stools with an interval of at least 1 week had been collected.

\section{Trial formula}

Both the FPF and the PF were supplied by Blédina. The PF was formulated to meet the nutritional needs of pre-term infants $^{(6,20)}$. The initial composition of the FPF was the same as that of the PF (Table 1). However, the manufacturing process included a fermentation step: $8 \mathrm{~h}$ at $37^{\circ} \mathrm{C}$ with two probiotic strains, B. breve C50 (Collection de l'Institut Pasteur, CIP I-2219) and $S$. thermophilus 065 (CIP I-1620), which were heat-inactivated at the end of the process This process added to the composition of the formula active bacterial metabolites such as non-digestible oligosaccharides. The packaging and the form were identical for each formula.

\section{Data collection}

Clinical data. Background information regarding pregnancy and neonatal parameters was collected, including sex, GA, birth weight, mode of delivery, neonatal distress, respiratory disease, infection, maternal steroid therapy, and antibiotic intake per partum and at birth. In order to evaluate the tolerance of the fermented formula, the following parameters were recorded twice a week until discharge: anthropometric parameters (weight, height and head circumference); gastrointestinal tolerance parameters (abdominal distension evaluated by inspection, gastric residuals, mucoid stool, loose stool, rectal bleeding and NEC). Drug administration and intake of the formula and mother's milk were also reported.

Stool collection. Faecal samples were collected each week from diapers and placed in sterile tubes. The tube for culture analysis contained $0.5 \mathrm{ml}$ brain heart infusion broth with $15 \%$ glycerol as a cryoprotective agent. All samples were immediately frozen after collection and kept at $-80^{\circ} \mathrm{C}$ until analysis. Data analyses were performed with the dietary groups coded, and the code was not broken until all analyses had been completed.

\section{Microbiological analysis of faecal samples}

Culture method. Qualitative and quantitative analyses of faecal flora allowing the isolation, quantification and 
Table 1. Composition of the standard formula

\begin{tabular}{|c|c|}
\hline Nutrition information for $15 \%$ dilution & $100 \mathrm{ml}$ \\
\hline Energy (kJ) & 314 \\
\hline Protein $(\mathrm{g})$ & $2 \cdot 2$ \\
\hline Casein:whey ratio & $40: 60$ \\
\hline Carnitine (mg) & $1 \cdot 2$ \\
\hline Taurine (mg) & 5 \\
\hline Nucleotides (mg) & 3.5 \\
\hline Fat $(\mathrm{g})$ & $3 \cdot 7$ \\
\hline МCT $(20 \%, g)$ & 0.7 \\
\hline Linoleic acid (mg) & 464 \\
\hline$\alpha$-Linoleic acid (mg) & 85 \\
\hline $\mathrm{DHA}(\mathrm{mg})$ & 7.4 \\
\hline ARA (mg) & $11 \cdot 1$ \\
\hline Carbohydrates (total, g) & 8 \\
\hline Lactose (g) & 5.5 \\
\hline Maltodextrin (g) & $2 \cdot 7$ \\
\hline \multicolumn{2}{|l|}{ Minerals } \\
\hline $\mathrm{Na}(\mathrm{mg})$ & 28 \\
\hline $\mathrm{K}(\mathrm{mg})$ & 80 \\
\hline $\mathrm{Ca}(\mathrm{mg})$ & 84 \\
\hline $\mathrm{P}(\mathrm{mg})$ & 44 \\
\hline $\mathrm{Mg}(\mathrm{mg})$ & 5 \\
\hline $\mathrm{Cl}(\mathrm{mg})$ & 55 \\
\hline $\mathrm{Fe}(\mathrm{mg})$ & 1.2 \\
\hline $\mathrm{Zn}(\mathrm{mg})$ & 0.4 \\
\hline \multicolumn{2}{|l|}{ Vitamins } \\
\hline$A(I U)$ & $210^{*}$ \\
\hline $\mathrm{D}(\mathrm{IU})$ & $42 \dagger$ \\
\hline $\mathrm{E}(\mathrm{mg})$ & 0.8 \\
\hline $\mathrm{K}_{1}(\mathrm{mg})$ & $8 \cdot 4$ \\
\hline $\mathrm{B}_{1}$ (thiamin, $\mathrm{mg}$ ) & 0.04 \\
\hline $\mathrm{B}_{2}$ (riboflavin, $\mathrm{mg}$ ) & $0 \cdot 14$ \\
\hline $\mathrm{B}_{6}(\mathrm{mg})$ & 0.04 \\
\hline$B_{12}(\mu \mathrm{g})$ & 0.15 \\
\hline PP (niacin equivalent, mg) & $1 \cdot 1$ \\
\hline$C(\mathrm{mg})$ & 7 \\
\hline Pantothenic acid (mg) & 0.27 \\
\hline Folic acid $(\mu \mathrm{g})$ & 49 \\
\hline Biotin $(\mu \mathrm{g})$ & $1 \cdot 2$ \\
\hline Choline (mg) & 2 \\
\hline Inositol (mg) & $3 \cdot 7$ \\
\hline
\end{tabular}

MCT, medium-chain TAG; ARA, arachidonic acid; pp, protocol population. ${ }^{*} 63 \mu \mathrm{g}$ (expressed in SI units).

$\dagger 1.1 \mu \mathrm{g}$ (expressed in SI units)

identification of the main genera were performed by spreading dilutions of the stools on various media as described previously $^{(21)}$. Bacterial counts were expressed as the $\log _{10}$ colony-forming units/g of faeces, and the count threshold was $3 \log _{10}$ colony-forming units/g of faeces. Identification of bifidobacteria was performed upon macroscopic aspects of colonies, microscopic characteristics and PCR using primers specific for genus and species, as described previously ${ }^{(22)}$.

Culture-independent method. Total faecal DNA extraction and bacterial PCR-temporal temperature gel electrophoresis (PCR-TTGE) were performed as described previously ${ }^{(23)}$. The Dcode Universal Mutation Detection System (BioRad, Hercules, CA, USA) was used for sequence-specific separation of amplicons. Known bacterial strains were used to standardise band migration and gel curvature among different gels. PCR-TTGE profiles were analysed using Diversity Database $2.1^{\circledR}$ (Bio-Rad).

Bacterial diversity was evaluated through the number of bands and the maturation of infant microbiota through the distance between TTGE profiles, which was calculated by comparison with the sample collected the week before for each infant. Mean values of the distances were analysed to obtain a general picture of faecal microbiota maturation.

\section{Faecal TNF- $\alpha$, calprotectin and secretory IgA measurements}

Faecal TNF- $\alpha$ was measured using ELISA (TNF- $\alpha$ EASIA ${ }^{\circledR}$; Biosource, Nivelles, Belgium) adapted for faecal samples as described previously ${ }^{(24)}$. Faecal calprotectin concentration was measured using a commercial ELISA kit (Calprest ${ }^{\circledR}$; Eurospital, Trieste, Italy) according to the manufacturer's instructions. Faecal SIgA were determined by ELISA using Nunc-Immuno plates (Maxisorp F96; Nunc, Roskilde, Denmark). Plates were coated for $1 \mathrm{~h}$ at $37^{\circ} \mathrm{C}$ and incubated overnight at $4^{\circ} \mathrm{C}$ with an antibody $(1 \mathrm{mg} / \mathrm{l})$ to human secretory component (Dako, Glostrup, Denmark) in veronal buffer (25 mmol/1; pH 8.6). Non-specific binding sites were blocked using PBS, pH 7.2, containing $1 \%$ bovine serum albumin. Serial twofold dilutions of tested samples and purified human SIgA (Cappel ICN, Aurora, CO, USA) were added to the wells. The plates were incubated for $1 \mathrm{~h}$ at $37^{\circ} \mathrm{C}$ under agitation, then washed and incubated for $1 \mathrm{~h}$ under agitation with a horseradish peroxidase-conjugated rabbit antibody $(0.25 \mu \mathrm{g} / \mathrm{l})$ to human IgA (Dako). Addition of O-phenyl diamine triggered the reaction. Absorbance was read at $492 \mathrm{~nm}$, and quantitative results were determined by reference to standard curves.

\section{Statistical analyses}

The primary outcomes were to evaluate the tolerance of the experimental formula and its effect on the gut microbiota establishment, in particular the increase in bifidobacterial counts. Since no differences were expected in terms of anthropometrics, the sample size estimation was only based on the expected effect on bifidobacteria, i.e. a $2 \log$ increase in the FPF compared with the PF as observed with an oligosaccharide-supplemented formula ${ }^{(25)}$. To detect such a difference with $80 \%$ power and $5 \% \alpha$ risk, twenty-five patients per group were required. The secondary outcome was to evaluate the effects of the experimental formula on faecal SIgA as an immunological marker and on faecal TNF- $\alpha$ and calprotectin as inflammatory markers.

Neonatal characteristics and quantitative variables concerning microbiota analyses are reported as means and standard deviations. Qualitative variables are reported as frequencies and percentages. Analyses were performed as intent-to-treat population analysis. Comparisons between the groups were performed using Fisher's exact test for qualitative parameters and Wilcoxon's test for continuous variables. For analysis of the independent roles of GA, mode of delivery, antibiotic therapy, intake of mother's milk and diet (FPF or PF) on the number of colonised infants and bacterial colonisation levels, multivariate logistic and linear regression models were used, respectively. 


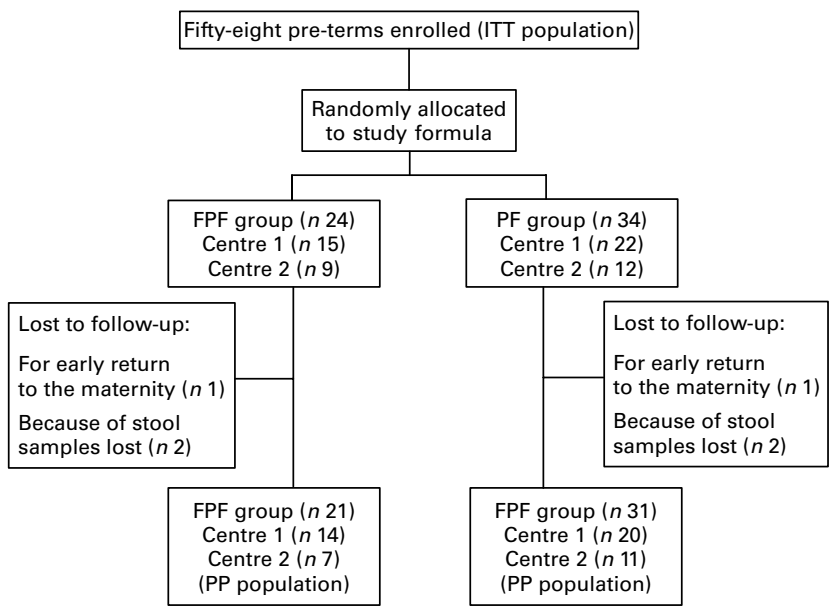

Fig. 1. Study flow chart. ITT, intent-to-treat; FPF, fermented pre-term formula; PF, pre-term formula.

\section{Results}

\section{Characteristics of the study population}

Written informed consent was obtained from the parents of fifty-eight pre-term infants (intent-to-treat population). Following a blind review, fifty-two infants remained as the per-protocol population, twenty-one in the FPF group and thirty-one in the PF group (Fig. 1). Among them, seven infants were followed up over 5 weeks, ten over 4 weeks, eighteen over 3 weeks and seventeen over 2 weeks. Within the intent-to-treat population (Table 2), there was no significant difference between the FPF and PF groups in terms of pregnancy and neonatal parameters. In centre 1, fifteen infants received their mother's milk, which was first administered at a median age of 7 (range $1-25$ ) d. The percentage of infants partially breast-fed was identical in both groups, with only six infants whose mother's milk intake was $50 \%$ or more from week 2 (two in the FPF group and four in the PF group). Infants receiving antibiotics (amoxicillin, broadspectrum cephalosporin, piperacillin, glycopeptide, aminoglycoside; used either alone or in combination) were treated from birth for suspected (eight infants) or confirmed (eight infants) infection with no differences between groups, and from day 4 for a nosocomial infection (one infant).

\section{Tolerance of the fermented formula: clinical characteristics of infants}

No differences in anthropometric data, i.e. catch-up growth, weight gain, recumbent length gain and head circumference gain, were found either between centres or between the feeding groups (Table 3). Abdominal distension was similar in the FPF (four infants) and PF (two infants) groups during weeks 1 and 2, but a significant difference occurred during weeks 3 and 4, with no abdominal distension in the FPF group and eight cases in the PF group $(P=0 \cdot 016)$. Gastric residuals ranged from 0 to $14.6 \%$, with no significant difference between the groups (mean values $2.5 \%$ in the FPF group $v$. $1.7 \%$ in the PF group). No differences in the stool patterns were observed between the two feeding groups. One infant fed the PF had rectal bleeding and was successfully treated for a suspected NEC (Bell's stage Ib). No adverse effects were observed throughout the study.

Table 2. Characteristics of the intent-to-treat population

(Mean values or number of infants and standard deviations)

\begin{tabular}{|c|c|c|c|c|c|c|c|}
\hline & \multicolumn{3}{|c|}{ FPF group ( $n$ 24) } & \multicolumn{3}{|c|}{ PF group ( $n$ 34) } & \multirow[b]{2}{*}{$P^{\star}$} \\
\hline & $n$ & Mean & SD & $n$ & Mean & SD & \\
\hline \multicolumn{8}{|l|}{ Sex } \\
\hline Boy & 15 & & & 16 & & & 0.29 \\
\hline Girl & 9 & & & 18 & & & \\
\hline \multicolumn{8}{|l|}{ Birth type } \\
\hline Single & 18 & & & 20 & & & 0.27 \\
\hline Twins & 6 & & & 14 & & & \\
\hline Gestational age (weeks) & & 33.5 & $1 \cdot 3$ & & $33 \cdot 4$ & $1 \cdot 1$ & 0.86 \\
\hline Birth weight $(\mathrm{g})$ & & 1912 & 354 & & 1926 & 386 & 0.89 \\
\hline Intra-uterine growth retardation & 4 & & & 5 & & & 1 \\
\hline \multicolumn{8}{|l|}{ Mode of delivery } \\
\hline Vaginal delivery & 13 & & & 15 & & & 0.60 \\
\hline Caesarean section & 12 & & & 18 & & & \\
\hline Maternal corticosteroids & 13 & & & 15 & & & 0.23 \\
\hline \multicolumn{8}{|l|}{ Maternal anti-biotherapy } \\
\hline Antenatal & 5 & & & 10 & & & 0.51 \\
\hline Per partum & 11 & & & 15 & & & 1 \\
\hline Neonatal distress & 1 & & & 1 & & & 1 \\
\hline Respiratory distress & 4 & & & 11 & & & 0.31 \\
\hline Neonatal anti-biotherapy & 8 & & & 9 & & & 0.56 \\
\hline Duration (d) & & 4.5 & $3 \cdot 6$ & & 5.6 & $2 \cdot 5$ & 0.49 \\
\hline Postnatal day of inclusion & & 1.8 & 1.2 & & 1.5 & 0.9 & 0.23 \\
\hline Mother's milk intake & 6 & & & 10 & & & 0.80 \\
\hline
\end{tabular}

FPF, fermented pre-term formula; PF, pre-term formula.

* There were no significant differences. 
Table 3. Anthropometric data of the intent-to-treat population (Mean values and standard deviations)

\begin{tabular}{|c|c|c|c|c|c|}
\hline & \multicolumn{2}{|c|}{$\begin{array}{l}\text { FPF group } \\
\quad(n 24)\end{array}$} & \multicolumn{2}{|c|}{$\begin{array}{l}\text { PF group } \\
\quad(n 34)\end{array}$} & \multirow[b]{2}{*}{$P^{*}$} \\
\hline & Mean & $S D$ & Mean & SD & \\
\hline Catch-up of birth weight (d) & 9 & 3 & 10 & 4 & NS \\
\hline $\begin{array}{l}\text { Weight gain during the } \\
\text { study period }(\mathrm{g} / \mathrm{d})\end{array}$ & 15 & 10 & 17 & 8 & NS \\
\hline $\begin{array}{l}\text { Length gain during the } \\
\text { study period (cm/week) }\end{array}$ & 0.8 & 0.4 & 0.7 & 0.5 & NS \\
\hline $\begin{array}{l}\text { Head circumference gain during } \\
\text { the study period ( } \mathrm{cm} / \text { week) }\end{array}$ & 0.8 & 0.4 & 0.7 & 0.3 & NS \\
\hline
\end{tabular}

FPF, fermented pre-term formula; PF, pre-term formula

* There were no significant differences.

\section{Establishment of the gut microbiota using culture}

Statistical analyses did not show any significant difference in bacterial colonisation between the centres. In the 151 faecal samples analysed (sixty-two from the FPF group and eightynine from the PF group), the number of colonised infants and the bacterial colonisation levels did not differ between the feeding groups throughout the study (Fig. 2). Most infants were rapidly colonised by staphylococci, mainly coagulasenegative. No more than two-thirds of all infants were colonised by enterococci and/or enterobacteria during the first week, with the percentage increasing throughout the study. Enterobacteria species were mainly Escherichia coli, Klebsiella sp. and Enterobacter cloacae, with no differences between the groups (Table 4). Bacteroides colonised a limited number of infants. By contrast, colonisation by clostridia was more frequent, increasing throughout the study up to $100 \%$ at the fourth week (see Table 4 for isolated species). Lactobacilli were seldom isolated.

Concerning the bifidobacterial colonisation, the number of colonised infants and the colonisation levels did not differ between the groups (Fig. 2). Bifidobacteria were isolated in five infants in the FPF group (24\%) and in ten infants in the PF group (32\%) at a mean level of 7.5 and $6.9 \log _{10}$ colonyforming units/g, respectively. Although not significant, mean bifidobacterial levels were higher in infants fed the FPF compared with those fed the PF $\left(7.5 \log _{10} v \cdot 6 \cdot 6 \log _{10}\right.$, respectively), but species were not different between the groups (Table 4). Antibiotics given either per partum or to the neonates had no significant influence. However, except for week 1 in the FPF group, maternal or neonatal antibiotics decreased bifidobacterial levels by an average of $2 \log _{10}$ whatever the type of feeding (Fig. 3). The only perinatal factor significantly affecting the incidence of bifidobacterial colonisation $(P=0.0064)$ and its levels $(P=0.03)$ was GA; no infants born at a GA under 33 weeks were colonised, which is concordant with what we have reported previously ${ }^{(22)}$. There was no difference in the effect of the type of feeding in infants born before 33 weeks GA compared with infants born at this GA or more, except for clostridia. Indeed, clostridial colonisation was significantly delayed in infants born at 33 weeks GA or more and fed the FPF, with none of these infants colonised during the first week of life by contrast with those fed the PF or those born at less than 33 weeks GA whatever the mode of feeding.

\section{Establishment of the gut microbiota using temporal temperature gel electrophoresis according to the feeding group}

TTGE was performed on eighty-seven faecal samples (thirtytwo from the FPF group and fifty-five from the PF group). The band set contained a total of eighty-six distinct bands, as shown for seven infants in Fig. 4. The number of bands/ sample varied from one to fourteen and did not increase from week 1 to week 3 (mean 6.1 (sD 3.1) to 7.8 (sD 3.3)/ sample). The distance values between the band patterns were very high, with a mean of 77 (SD 21)\% between weeks 1 and 2 and 73 (SD 24) \% between weeks 2 and 3. A band corresponding to bifidobacteria was detected in the faecal samples of five infants in the FPF group and six infants in the PF group. Neither GA nor the type of feeding influenced significantly the profile patterns throughout the study.

\section{Faecal TNF- $\alpha$, calprotectin and secretory IgA}

Overall, 145 faecal samples were tested. TNF- $\alpha$ was detected in four samples (four infants) in the FPF group (median 41 (range $31-57) \mathrm{pg} / \mathrm{g}$ ) and nine samples (seven infants) in the PF group (median 60 (range 30-155) pg/g), with no significant differences between the groups.

Faecal calprotectin was detectable in all samples with high inter- and intra-individual variation (median 257 (range 161240) $\mu \mathrm{g} / \mathrm{g}$ ) and no relationship with GA. A downward trend in calprotectin levels was observed in the FPF group throughout the study period. This decrease in calprotectin levels was significant in the FPF group $(P=0.01)$ from week 3 (Fig. 5). Differences between the two groups were not significant in infants receiving antibiotics, as opposed to infants without any antibiotics $(P=0.048)$. Partial breast-feeding did not influence calprotectin levels whatever the group. Faecal SIgA levels were detectable in all samples, with high inter-individual variation. No differences were observed over the entire study period between infants fed only the PF (median 12 (range $1-350) \mu \mathrm{g} / \mathrm{g}$ ) or the FPF (median (27 (range 1-474) $\mu \mathrm{g} / \mathrm{g}$ ) (Fig. 6(a)). As expected, SIgA of partially breast-fed infants were higher in both the feeding groups. Interestingly, in infants partially breast-fed, SIgA was significantly higher $(P=0.02)$ from week 2 in infants fed the FPF (median: 3038 (range 1225-6040) $\mu \mathrm{g} / \mathrm{g}$ compared with those fed the PF (median 1473 (range 30-2655) $\mu \mathrm{g} / \mathrm{g}$ ) (Fig. 6(b)).

\section{Discussion}

This trial tested the tolerance and the effect on the bacterial colonisation of a FPF without live bacteria that had never previously been tested in pre-term infants. It was found that the FPF was well tolerated, and similarly to the standard PF, weight gain, head circumference and recumbent length did not differ from reference chart values.

Such good tolerance of prebiotic supplementation of the formula has previously been reported in pre-term infants in 
(b)

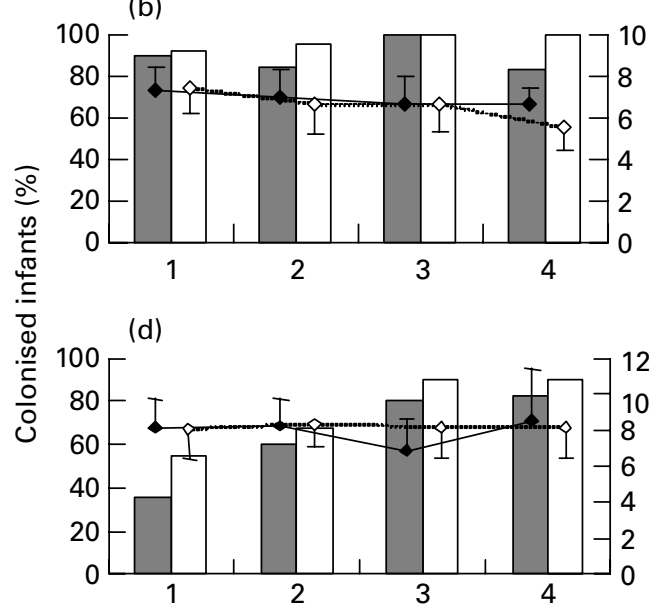

(f)

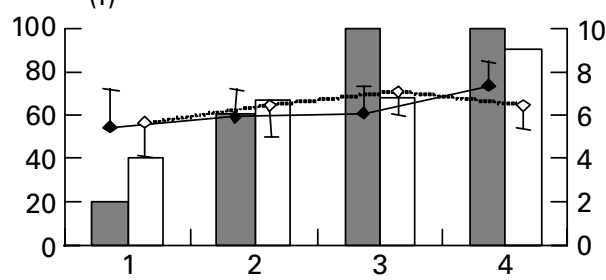

(c)

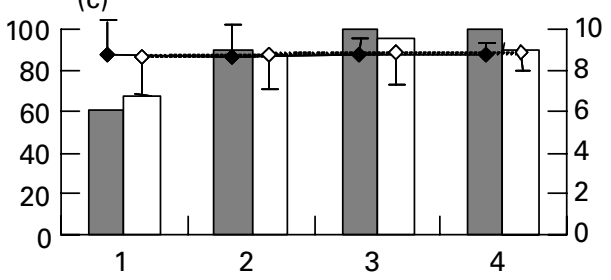

(e)

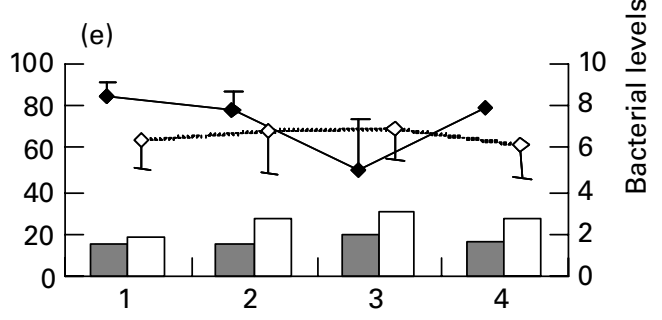

(g)

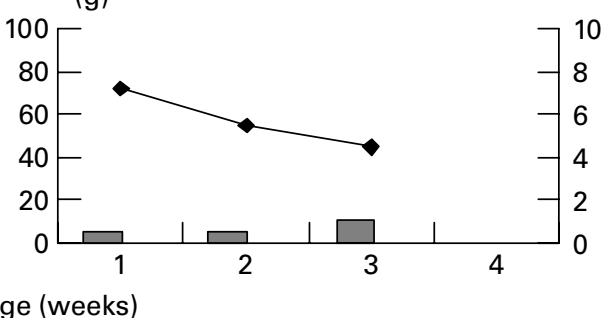

Fig. 2. Microbiota colonisation during the first 4 weeks of life in the fermented pre-term formula (FPF) and pre-term formula (PF) groups. Bar graphs indicate colonised infants (\%): FPF group ( $\square$ ), PF group ( $\square$ ). Line graphs indicate mean levels of colonisation, with standard deviation represented by vertical bars: FPF group $(\neg)$, PF group $(\diamond-)$. (a) Bifidobacterium, (b) Staphylococcus, (c) Enterococcus, (d) Enterobacteria, (e) Bacteroides, (f) Clostridium, (g) Lactobacillus. CFU, colony-forming unit.

four small clinical trials ${ }^{(11)}$. This is of great importance, since reduction in weight gain and/or growth can be detrimental for pre-term infants. Moreover, in the present study, intestinal tolerance tended to be improved in infants fed the FPF, as shown by less-frequent abdominal distension observed after 2 weeks of enteral feeding. This effect might be compared with the beneficial effects on intestinal tolerance observed in pre-term infants fed a probiotic-supplemented pre-term formula ${ }^{(26)}$. However, interpreting these data are difficult, as it may indicate a decrease in gas production, an increase in motility or a decrease in mucosal inflammation, although it is of note that no effect on the gastric residual volume was observed.

No bifidogenic effect was observed with this formula, which contrasts with that found for full-term infants fed a similar formula from the first month of feeding ${ }^{(17)}$. However, in the latter study, bifidobacteria were detected in all infants from the beginning of the survey. Likewise, prebiotic activity of a preterm formula containing oligosaccharides has been observed in pre-term infants in two clinical trials ${ }^{(11)}$, but, again, these infants harboured high levels of bifidobacteria from inclusion. In a recent study, no increase in the number of colonised infants and a non-significant increase in bifidobacterial levels have been observed in pre-term infants receiving a dietary supplement of lactobacilli and fructo-oligosaccharides and displaying a low incidence of bifidobacterial colonisation at enrolment ${ }^{(27)}$. This tends to support that, if bifidobacteria at baseline are absent or at low levels, no metabolites or other molecules can have bifidogenic properties. However, in infants colonised by bifidobacteria, the average bifidobacterial levels were about 1 log higher in infants fed the FPF compared with those fed the PF. Although not statistically significant, such an increase could have some biological effects. The lack of a prebiotic effect could also be due to a too low dose of oligosaccharides produced during the fermentation process, since a dose-dependent prebiotic effect has previously been demonstrated with a formula supplemented with two doses of a mixture of oligosaccharides ${ }^{(28)}$. The role 
Table 4. Bacterial species belonging to enterobacteria, clostridia and bifidobacteria isolated in fifty-two pre-term infants (per-protocol population)

\begin{tabular}{lccl}
\hline & $\begin{array}{c}\text { FPF group } \\
(n \text { 21) }\end{array}$ & $\begin{array}{c}\text { PF group } \\
(n \text { 31) }\end{array}$ & $P^{*}$ \\
\hline Bifidobacteria & & & \\
B. bifidum & $2 \dagger$ & 3 & 1 \\
B. breve & 0 & 2 & 0.51 \\
B. longum & 2 & 2 & 1 \\
B. pseudocatenulatum & 1 & 3 & 0.64 \\
B. animalis subsp. lactis & 0 & 2 & 0.51 \\
B. adolescentis & 0 & 1 & 1 \\
Enterobacteria & & & \\
Escherichia coli & 7 & 15 & 0.39 \\
Klebsiella sp. & 4 & 6 & 1 \\
Enterobacter cloacae & 7 & 8 & 0.76 \\
Clostridia & & & \\
C. perfringens & 9 & 12 & 0.78 \\
C. difficile & 6 & 12 & 0.55 \\
C. butyricum & 4 & 5 & 1 \\
C. paraputrificum & 1 & 3 & 0.63 \\
\hline
\end{tabular}

FPF, fermented pre-term formula; PF, pre-term formula.

* There were no significant differences.

tNumber of colonised infants.

of macronutrients should not be overlooked, but the formula composition was similar to that tested in full-term infants ${ }^{(17)}$.

Since we have previously reported that bifidobacterial colonisation did not occur in infants born at a GA less than 33 weeks $^{(22)}$, the effect of the FPF could be dependent on GA. However, we did not find any difference in the effect of the formula either in infants born before a GA of 33 weeks or in those born after a GA of 33 weeks or more, except for clostridia, which did not colonise any infant born at GA of 33 weeks or more and fed the FPF during the first week of life. This delay could be due to either the combination of bifidobacterial colonisation and the presence of active metabolites or an indirect effect of the FPF on the innate intestinal immune system or both. Clinical beneficial effects of such delay are unknown. However, clostridia are strongly involved in $\mathrm{NEC}^{(29)}$, and early intestinal colonisation by this genus has been linked to $\mathrm{NEC}^{(30)}$.

Additionally, we confirmed the abnormal gut bacterial colonisation and the low bacterial diversity in pre-term infants described in other studies ${ }^{(1,31-34)}$, with no significant differences between the two feeding groups. Colonisation by aerobes occurred very early, especially for staphylococci,

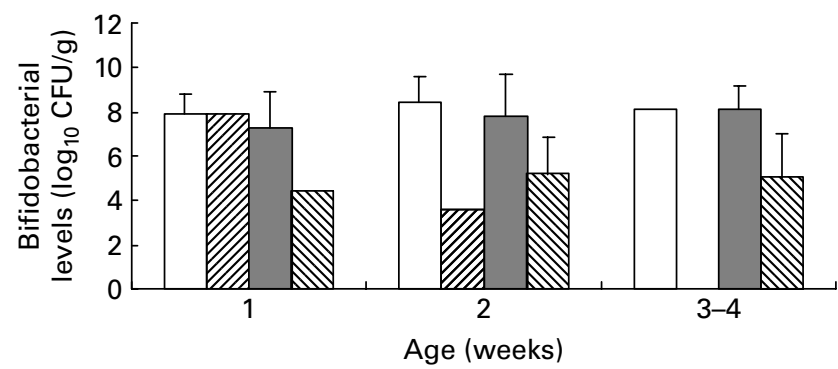

Fig. 3. Effect of the neonatal anti-biotherapy on bifidobacterial levels. Values are means, with standard deviations represented by vertical bars. Fermented pre-term formula group, without antibiotics ( $\square$ ), with antibiotics $(\square)$, pre-term formula group, without antibiotics $(\square)$, with antibiotics $(\mathbb{\Xi})$. CFU, colony-forming unit.

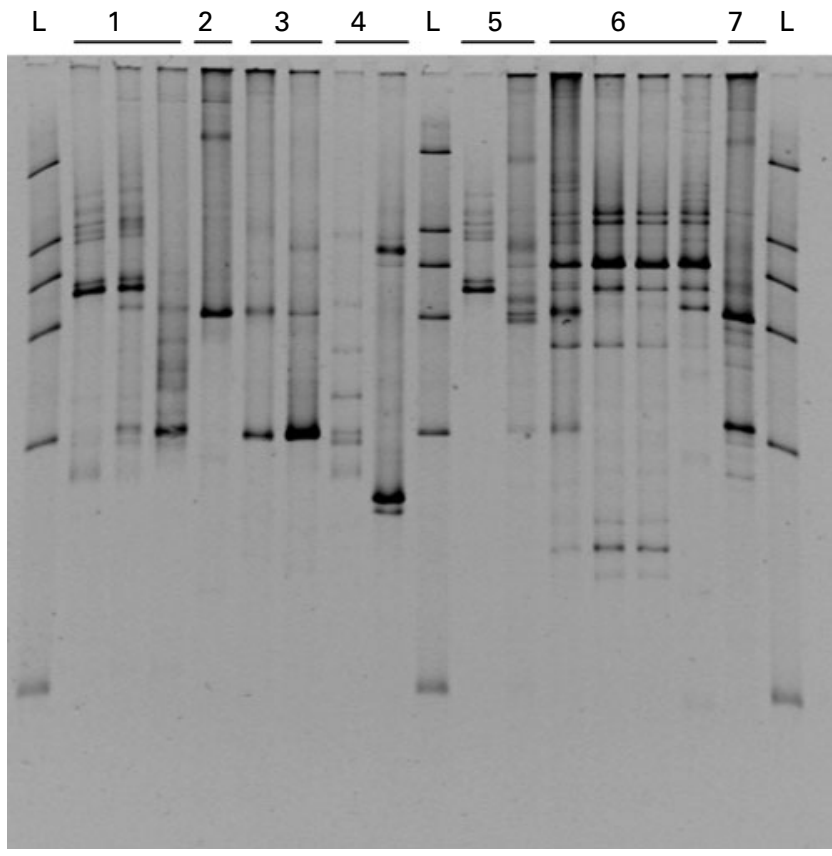

Fig. 4. PCR-temporal temperature gel electrophoresis profiles of faecal samples from seven pre-term infants. L, bacterial standardisation ladder, from top to bottom: Bacteroides sp., Prevotella sp., Enterococcus faecium, Staphylococcus epidermidis, Escherichia coli and Bifidobacterium longum.

whereas colonisation by anaerobes, especially bifidobacteria as highlighted earlier, was delayed. The high incidence and levels of clostridia described in the present study have rarely been reported in the literature ${ }^{(35)}$.

Gut inflammatory status and immune mucosal response of pre-term infants were investigated in the present study through the measurement of faecal calprotectin, TNF- $\alpha$ and SIgA. Although the proportion of infants with detectable TNF- $\alpha$ was too low to sufficiently allow powerful statistical analysis, data showed that the FPF was associated with altered calprotectin and SIgA levels. High levels of faecal calprotectin have long been associated with inflammatory gastrointestinal diseases in children and adults but appear to also occur in healthy neonates ${ }^{(36-38)}$. These high levels may relate to a

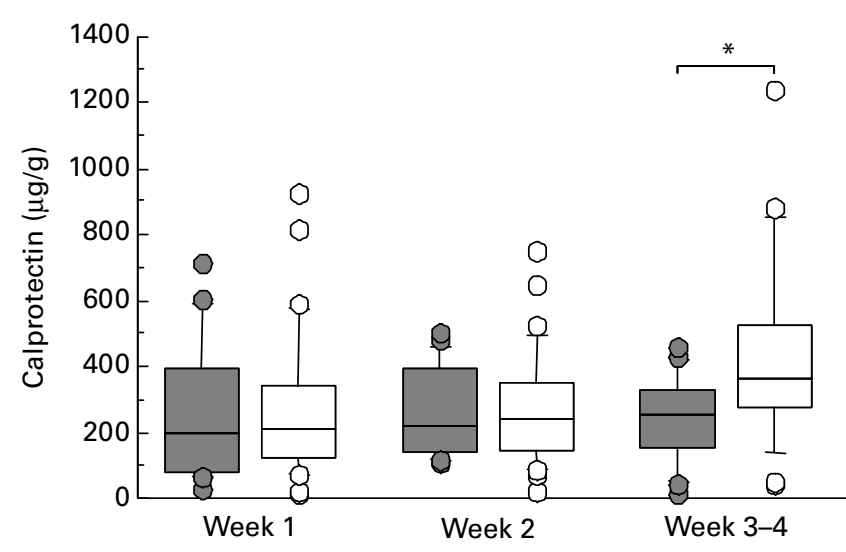

Fig. 5. Boxplots of faecal calprotectin during the first month: fermented pre-term formula group $(\square)$, pre-term formula (PF) group ( $\square$ ). Values are means, with standard deviations represented by vertical bars. * Mean values were significantly different from those of the PF group $(P=0.001)$. 

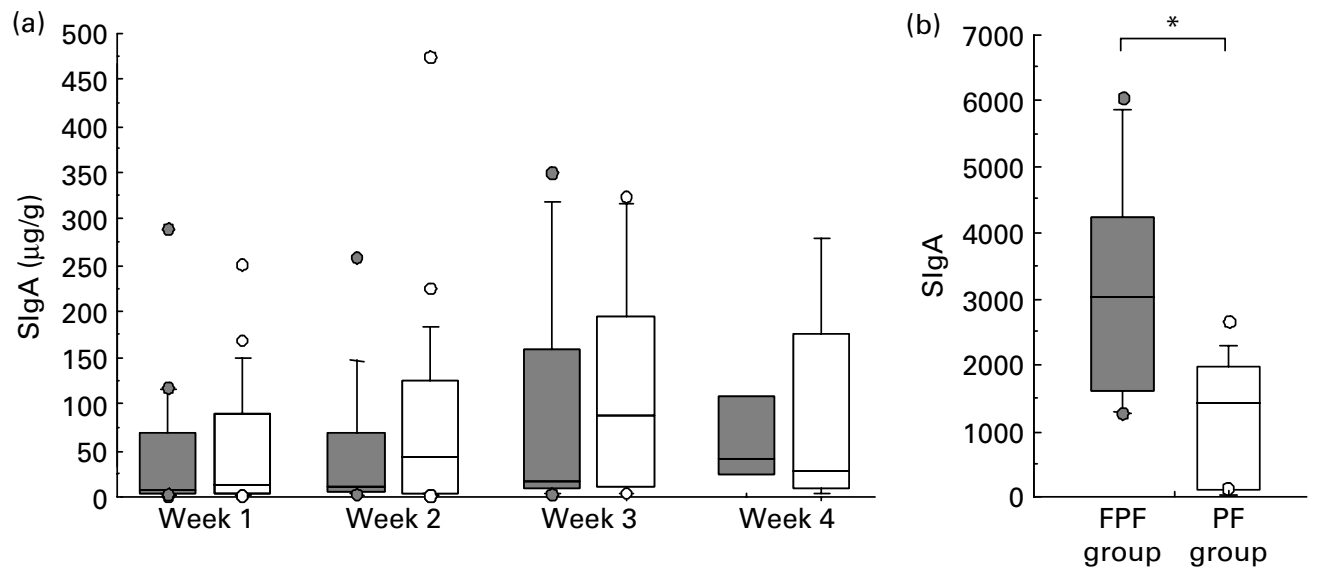

Fig. 6. Boxplots of faecal secretory IgA (SIgA) levels during the first month: fermented pre-term formula (FPF) group ( $\square$ ), pre-term formula (PF) group ( $\square$ ). Values are means, with standard deviations represented by vertical bars. (a) Infants receiving the formulas exclusively. (b) Infants partially breast-fed at weeks 2 and 3 . * Mean values were significantly different $(P=0.05)$.), pre-term formula (PF) group ( $\square$ ). Values are means, with standard deviations represented by vertical bars. (a) Infants receiving the formulas exclusively. (b) Infants partially breast-fed at weeks 2 and 3 . * Mean values were significantly different $(P=0.05)$.

physiological inflammation at birth linked to the presence of numerous dietary and bacterial antigens ${ }^{(39)}$. Calprotectin levels were also found to be high in the present study with wide inter-individual variations and no relationship with GA, as reported previously in healthy full-term and pre-term infants ${ }^{(31,36-38,40)}$. After 2 weeks of feeding, infants from the FPF group had significantly lower faecal calprotectin levels than those of the PF group, suggesting that the FPF may contribute in attenuating the inflammatory response to dietary and bacterial antigens, as reported previously with Bifidobacterium lactis Bb12 supplementation ${ }^{(41)}$. This result could be linked to the reduced prevalence of abdominal distension observed from week 3 in the FPF group.

SIgA play a central role in local immunity and participate in the mucosal intestinal barrier against pathogenic bacteria or viruses. SIgA appear to increase due to both mother's milk and the use of the fermented formula, suggesting a modulation of immunity, as demonstrated previously by a significant increase in SIgA after poliovirus vaccination in full-term infants fed the similar fermented formula without live bacteria ${ }^{(17)}$. Mohan et $a l .{ }^{(41)}$ reported such an increase in total faecal SIgA in pre-term infants supplemented early after birth with B. lactis Bb12 over a 3-week period. Both these data suggest a positive effect of probiotic/prebiotic supplementation on gut-associated lymphoid tissue maturation in pre-terms infants.

The effect that we observed needs to be assessed on a larger number of infants, and, if confirmed, the mechanisms by which the formula may reduce inflammation and promote local immune responses would deserve further investigation.

To conclude, the present study evaluated for the first time the effect on clinical tolerance, gut microbiota and mucosal immuno-inflammatory responses of a fermented pre-term infant formula without live bacteria. Clinical tolerance was similar to that of a classic pre-term formula, with no adverse effects. No bifidogenic effect was observed in contrast with the observation made with a similar formula in full-term infants. Thus, according to our findings, prebiotic molecules may not influence bifidobacterial colonisation in pre-term infants displaying poor bifidobacterial colonisation. However, this formula may attenuate inflammatory responses to dietary and bacterial antigens and promote mucosal immunity when associated with breast-feeding, thus improving gastrointestinal tolerance. These effects could be part of a strategy of minimal enteral feeding to accelerate the maturation of gastrointestinal function.

\section{Acknowledgements}

We thank Ester Pereira for technical assistance and Cecile Aubert-Jacquin and Danone Baby Nutrition Research, Villefranche-sur-Saône, for providing financial support for the present study. The authors' contributions were as follows: F. C., N. Kap., A.-J. W.-D., N. Kal., C. D. and M.-J. B. contributed to the study concept and design; F. C., P. S., B. L. and C. D. were responsible for the clinical data collection and faecal sample collection; A. S., F. M., N. Kap., L. F., A.-J. W.-D. and M.-J. B. conducted the microbiota and faecal marker analyses; V. V. performed the statistical analysis; F. C., A. S., N. Kap., F. M., A.-J. W.-D., B. L., N. Kal., C. D. and M.-J. B. were involved in the data interpretation and critical revision of the manuscript; F. C., N. Kap., C. D. and M.-J. B. assisted in drafting the manuscript; C. D. and M.-J. B. were responsible for the overall supervision of the study. None of the grant suppliers had any involvement in the analysis of the data or interpretation of the results. None of the authors had any conflicts of interests.

\section{References}

1. Magne F, Abély M, Boyer F, et al. (2006) Low species diversity and high diversity variability in feces of preterm infants as revealed by $16 \mathrm{~s}$ rDNA sequences and PCR-TTGE profiles. FEMS Microbiol Ecol 57, 128-138.

2. Szajewska H (2010) Probiotics and prebiotics in preterm infants: where are we? where are we going? Early Hum Dev 86, S81-S86.

3. Veereman-Wauters G (2005) Application of prebiotics in infant foods. Br J Nutr 93, Suppl. 1, S57-S60. 
4. Alfaleh K, Anabrees J \& Bassler D (2010) Probiotics reduce the risk of necrotizing enterocolitis in preterm infants: a meta-analysis. Neonatology 97, 93-99.

5. Deshpande G, Rao S, Patole S, et al. (2010) Updated metaanalysis of probiotics for preventing necrotizing enterocolitis in preterm neonates. Pediatrics 125, 921-930.

6. Agostoni C, Buonocore G, Carnielli VP, et al. (2010) Enteral nutrient supply for preterm infants: commentary from the European Society of Paediatric Gastroenterology, Hepatology and Nutrition Committee on Nutrition. J Pediatr Gastroenterol Nutr 50, 85-91.

7. Boyle RJ, Robins-Browne RM \& Tang ML (2006) Probiotic use in clinical practice: what are the risks? Am J Clin Nutr 83, 1256-1264

8. Ohishi A, Takahashi S, Ito Y, et al. (2010) Bifidobacterium septicemia associated with postoperative probiotic therapy in a neonate with omphalocele. J Pediatr 156, 679-681.

9. Roberfroid M (2007) Prebiotics: the concept revisited. J Nutr 137, 830S-837S.

10. Lomax AR \& Calder PC (2009) Probiotics, immune function, infection and inflammation: a review of the evidence from studies conducted in humans. Curr Pharm Des 15, 1428-1518.

11. Srinivasjois R, Rao S \& Patole S (2009) Prebiotic supplementation of formula in preterm neonates: a systematic review and meta-analysis of randomised controlled trials. Clin Nutr 28, 237-242.

12. Mihatsch WA, Hoegel J \& Pohlandt F (2006) Prebiotic oligosaccharides reduce stool viscosity and accelerate gastrointestinal transport in preterm infants. Acta Paediatr 95, 843-848.

13. Westerbeek EA, van den Berg JP, Lafeber HN, et al. (2010) Neutral and acidic oligosaccharides in preterm infants: a randomized, double-blind, placebo-controlled trial. $A m \mathrm{~J}$ Clin Nutr 91, 679-686.

14. Agostoni C, Goulet O, Kolacek S, et al. (2007) Fermented infant formulae without live bacteria. J Pediatr Gastroenterol Nutr 44, 392-397.

15. Menard S, Candalh C, Ben AM, et al. (2006) Stimulation of immunity without alteration of oral tolerance in mice fed with heat-treated fermented infant formula. J Pediatr Gastroenterol Nutr 43, 451-458.

16. Hoarau C, Lagaraine C, Martin L, et al. (2006) Supernatant of Bifidobacterium breve induces dendritic cell maturation, activation, and survival through a toll-like receptor 2 pathway. J Allergy Clin Immunol 117, 696-702.

17. Mullie C, Yazourh A, Thibault H, et al. (2004) Increased poliovirus-specific intestinal antibody response coincides with promotion of Bifidobacterium longum-infantis and Bifidobacterium breve in infants: a randomized, doubleblind, placebo-controlled trial. Pediatr Res 56, 791-795.

18. Thibault H, Aubert-Jacquin C \& Goulet O (2004) Effects of long-term consumption of a fermented infant formula (with Bifidobacterium breve c50 and Streptococcus thermophilus 065) on acute diarrhea in healthy infants. J Pediatr Gastroenterol Nutr 39, 147-152.

19. Indrio F, Ladisa G, Mautone A, et al. (2007) Effect of a fermented formula on thymus size and stool $\mathrm{pH}$ in healthy term infants. Pediatr Res 62, 98-100.

20. Hay WW Jr (2008) Strategies for feeding the preterm infant. Neonatology 94, 245-254.

21. Rougé C, Goldenberg O, Ferraris L, et al. (2010) Investigation of the intestinal microbiota in preterm infants using different methods. Anaerobe 16, 362-370.

22. Butel MJ, Suau A, Campeotto F, et al. (2007) Conditions of bifidobacterial colonization in preterm infants: a prospective analysis. J Pediatr Gastroenterol Nutr 44, 577-582.
23. Magne F, Hachelaf W, Suau A, et al. (2006) A longitudinal study of infant faecal microbiota during weaning. FEMS Microbiol Ecol 58, 563-571

24. Kapel N, Matarazzo P, Haouchine D, et al. (1999) Fecal tumor necrosis factor alpha, eosinophil cationic protein and IgE levels in infants with cow's milk allergy and gastrointestinal manifestations. Clin Chem Lab Med 37, 29-32.

25. Boehm G, Lidestri M, Casetta P, et al. (2002) Supplementation of a bovine milk formula with an oligosaccharide mixture increases counts of faecal bifidobacteria in preterm infants Arch Dis Child Fetal Neonatal Ed 86, F178-F181.

26. Indrio F, Riezzo G, Raimondi F, et al. (2009) Prebiotics improve gastric motility and gastric electrical activity in preterm newborns. J Pediatr Gastroenterol Nutr 49, 258-261.

27. Underwood MA, Salzman NH, Bennett SH, et al. (2009) A randomized placebo-controlled comparison of 2 prebiotic/probiotic combinations in preterm infants: impact on weight gain, intestinal microbiota, and fecal short-chain fatty acids. J Pediatr Gastroenterol Nutr 48, 216-225.

28. Moro G, Minoli I, Mosca M, et al. (2002) Dosage-related bifidogenic effects of galacto- and fructooligosaccharides in formulafed term infants. J Pediatr Gastroenterol Nutr 34, 291-295.

29. Waligora-Dupriet AJ, Dugay A, Auzeil N, et al. (2005) Evidence for clostridial implication in necrotizing enterocolitis through bacterial fermentation in a gnotobiotic quail model. Pediatr Res 58, 629-635.

30. De La Cochetière MF, Piloquet H, Des Robert C, et al. (2004) Early intestinal bacterial colonization and necrotizing enterocolitis in premature infants: the putative role of Clostridium. Pediatr Res 56, 1-5.

31. Rougé C, Piloquet H, Butel MJ, et al. (2009) Oral supplementation with probiotics in very-low-birth-weight preterm infants: a randomized, double-blind, placebo-controlled trial. Am J Clin Nutr 89, 1828-1835.

32. Gewolb IH, Schwalbe RS, Taciak VL, et al. (1999) Stool microflora in extremely low birthweight infants. Arch Dis Child Fetal Neonatal Ed 80, F167-F173.

33. Schwiertz A, Gruhl B, Lobnitz M, et al. (2003) Development of the intestinal bacterial composition in hospitalized preterm infants in comparison with breast-fed, full-term infants. Pediatr Res 54, 393-399.

34. Roudiere L, Jacquot A, Marchandin H, et al. (2009) Optimized PCR-temporal temperature gel electrophoresis compared to cultivation to assess diversity of gut microbiota in neonates. J Microbiol Methods 79, 156-165.

35. Westerbeek EA, van den BA, Lafeber HN, et al. (2006) The intestinal bacterial colonisation in preterm infants: A review of the literature. Clin Nutr 25, 361-368.

36. Campeotto F, Kalach N, Lapillonne A, et al. (2007) Time course of faecal calprotectin in preterm newborns during the first month of life. Acta Paediatr 96, 1531-1533.

37. Campeotto F, Butel MJ, Kalach N, et al. (2004) High faecal calprotectin concentrations in newborns infants. Arch Dis Child Fetal Neonatal Ed 89, F353-F355.

38. Savino F, Castagno E, Calabrese R, et al. (2009) High faecal calprotectin levels in healthy, exclusively breast-fed infants Neonatology 97, 299-304.

39. Rougé C, Butel MJ, Piloquet H, et al. (2010) Fecal calprotectin excretion in preterm infants during the neonatal period. PLoS One 5, e11083.

40. Yang Q, Smith PB, Goldberg RN, et al. (2008) Dynamic change of fecal calprotectin in very low birth weight infants during the first month of life. Neonatology 94, 267-271.

41. Mohan R, Koebnick C, Schildt J, et al. (2008) Effects of Bifidobacterium lactis supplementation on body weight, fecal $\mathrm{pH}$, acetate, lactate, calprotectin and IgA in preterm infants. Pediatr Res 64, 418-422. 